\title{
Ausência de Conexão Atrioventricular à Direita. Apresentação Morfológica e Clínica quando o Ventrículo Principal é Morfologicamente Direito
}

\author{
José Fernando Cavalini, Vera Demarchi Aiello, Munir Ebaid
}

São Paulo, SP

Ausência de conexão atrioventricular à direita com o ventrículo dominante, morfologicamente direito, colocado à esquerda, em conexão com o átrio esquerdo, é uma rara situação. Os autores apresentam cinco casos com esta disposição morfológica, salientando os aspectos anatômicos e clínicos, bem como a importância da nomenclatura baseada na análise segmentar seqüencial.

\section{Absence of the Right Atrioventricular Connection. Morphological and Clinical Presentation when the Dominant Ventricle is Morphologically Right.}

Absent right atrioventricular connection, with the left atrium connected to a dominant left-sided morphologically right ventricle is a rare situation. We report five cases with these anatomical features, with emphasis on the morphological and clinical aspects.

Although this combination of lesions is very uncommon, the sequential segmental analysis can easily provide an accurate description of the anomaly.

Arq Bras Cardiol, volume 71 (n 6), 793-796, 1998

Ausência de conexão atrioventricular direita, freqüentemente, acompanha-se de concordância atrioventricular à esquerda, com o átrio esquerdo (AE) conectado ao ventrículo morfologicamente esquerdo, dominante à esquerda (a chamada atresia tricúspide clássica) com concordância ventriculoarterial ou ainda, menos freqüentemente, com discordância ventriculoarterial. Raramente, encontramos situações onde o ventrículo morfologicamente direito está conectado ao AE, com relação ventriculoarterial predominantemente do tipo dupla via de saída do ventrículo direito(VD) ${ }^{1}$.

O propósito deste trabalho foi de apresentar esta rara cardiopatia congênita, com ampla discussão sobre a nomenclatura anatômica, salientando-se a importância da análise segmentar seqüencial neste tipo de associação de defeitos, para evitar confusão na sua interpretação. São relatados cinco casos, por nós estudados, de ausência de conexão atrioventricular direita com o ventrículo morfologicamente

Instituto do Coração do Hospital das Clínicas - FMUSP.

Correspondência: José Fernando Cavalini - Incor - Av. Dr. Enéas C. Aguiar 44 05403-000 - São Paulo, SP.

Recebido para publicação em 13/2/98

Aceito em 14/10/98 direito dominante situado à esquerda conectado ao $\mathrm{AE}$, sendo que quatro deles já foram previamente relatados ${ }^{2}$.

\section{Relato dos casos}

Todos os cinco pacientes apresentavam semiologia clínica e achados de exames complementares semelhantes. A cianose estava presente em todos. As idades, por ocasião da apresentação, e o sexo estão apresentados na tabela. Ao exame do precordio, apresentavam hiperfonese do $1^{\circ}$ ruído cardíaco na área mitral (ápex cardíaco), mais intensamente do que na área tricúspide, com a $2^{\mathrm{a}}$ bulha hiperfonética na área pulmonar irradiando para a área mitral mais do que para a área tricúspide. Nos dois pacientes com dextrocardia, um em situs solitus e o outro em ambíguo (isomerismo atrial esquerdo), a intensidade dos ruídos cardíacos era mais acentuada na borda esternal direita baixa (área mitral) do que na linha hemiclavicular direita (área tricúspide).

Os aspectos eletrocardiográfico e vetocardiográfico foram semelhantes também nos cinco casos, mostrando sobrecarga do ventrículo colocado à esquerda, com rotação anti-horária e para a esquerda no plano horizontal da alça vetocardiográfica da despolarização ventricular (fig. 1). A radiografia de tórax mostrava aspecto sugestivo de aorta em 


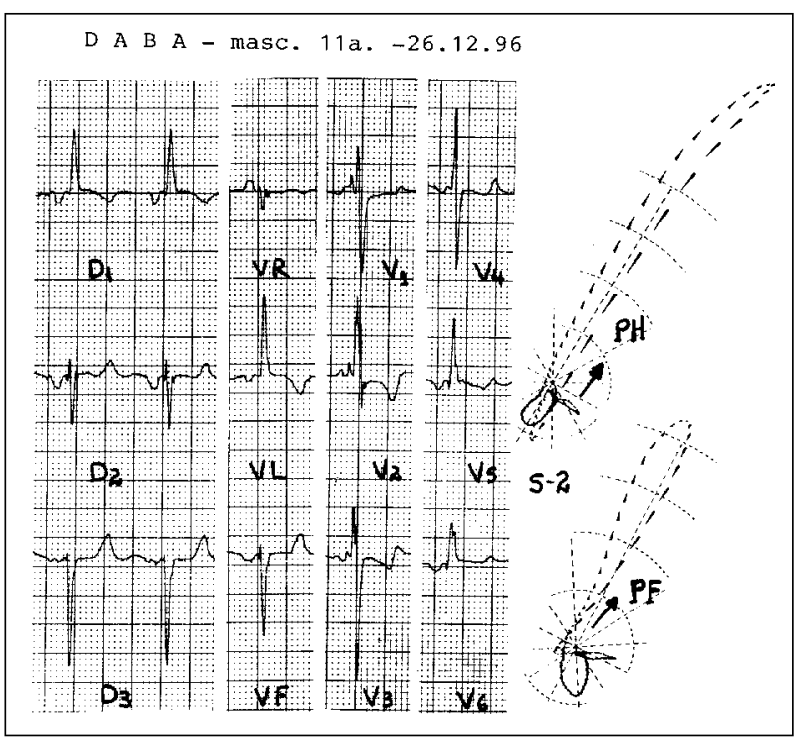

Fig. 1 - Eletrocardiograma e vetocardiograma do caso $n^{\circ} 4$ mostrando sobrecarga do ventrículo situado à esquerda, com rotação anti-horária e para a esquerda no plano horizontal.

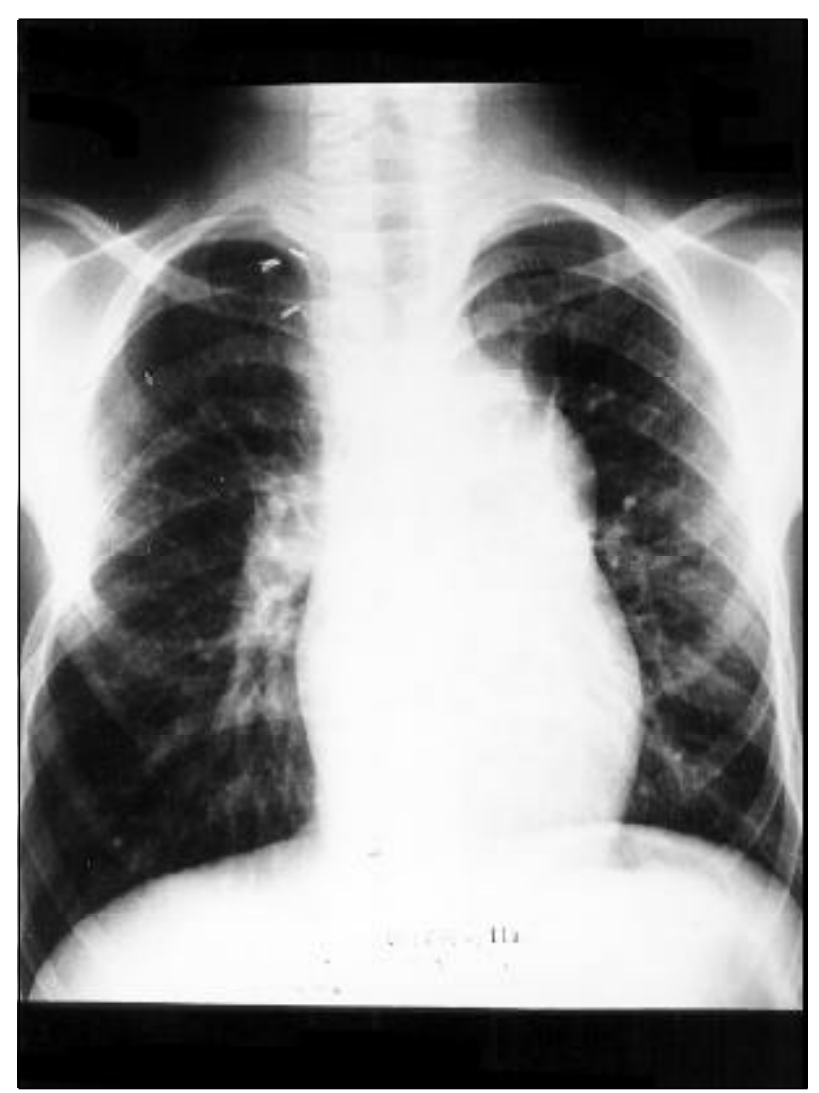

Fig. 2 - Radiografia de tórax do caso $\mathrm{n}^{\circ} 4$ com contorno superior à esquerda sugestivo de aorta em posição de "transposição corrigida das grandes artérias".

posição de "transposição corrigida das grandes artérias" nos três casos com situs solitus e levocardia (fig. 2). O ecocardiograma em todos os pacientes e o estudo angiográfico em quatro deles, mostraram a ausência de conexão atrioventricular à direita, com o ventrículo dominante morfologi- camente direito à esquerda em conexão com o AE, e a relação ventriculoarterial do tipo dupla via de saída deste VD em todos os casos. Quatro pacientes foram considerados para tratamento cirúrgico, sendo que em um foi realizada a anastomose cavopulmonar total, com resultado favorável (já em oito anos de evolução pós-operatória). Dois deles foram submetidos a anastomose de Blalock-Taussig, e no único a apresentar hipertensão pulmonar, realizou-se bandagem do tronco pulmonar e atrio-septectomia cirúrgica. Dois pacientes evoluíram para óbito e os achados necroscópicos foram: caso 1 - situs solitus, com a ponta do coração à direita; conexão venosa sistêmica e pulmonar normais; ausência da conexão atrioventricular à direita, com comunicação interatrial tipo fossa oval; o AE era conectado com o ventrículo dominante morfologicamente direito, situado à esquerda (topologia de mão esquerda), através de valva atrioventricular morfologicamente tricúspide; aorta emergindo deste ventrículo, anteriormente a um tronco pulmonar atrésico (fig. 3). Presença de um VE rudimentar à direita, posterior e inferior, representado apenas pela sua porção trabecular apical. Arco aórtico à esquerda, e canal arterial pérvio; caso 2 - isomerismo atrial esquerdo, com a ponta do coração à direita; veia cava superior à esquerda conectada com o átrio à esquerda, assim como as veias supra-hepáticas; o átrio à direita recebia as veias pulmonares e havia ausência de conexão atrioventricular à direita; septectomia atrial cirúrgica ao nível da fossa oval; valva atrioventricular com morfologia de tricúspide, conectada ao VD dominante situado à esquerda (topologia de mão esquerda), de onde emergiam ambos os vasos arteriais. Havia um infundíbulo muscular completo subpulmonar anteriormente, com aorta posterior e à esquerda (fig. 4), e seu arco sobre o brônquio direito. O canal arterial era pérvio, e o VErudimentar à direita era inferior e posterior.

\section{Discussão}

Ausência de conexão atrioventricular direita com o VD dominante situado à esquerda, em conexão com o AE, é uma situação rara. Cinco casos são apresentados com esta disposição, sendo quatro deles em situs solitus, todos com estenose ou atresia pulmonar e um com isomerismo atrial esquerdo e hipertensão pulmonar. Todos apresentavam dupla via de saída do VD.

Uma análise clínica através dos elementos de exame físico, eletro e vetocardiograma, além da radiografia de tórax, nos levaram à suspeita diagnóstica. A maior intensidade do $1^{\circ}$ ruído cardíaco no ápex cardíaco (área mitral) e do $2^{\circ}$ ruído cardíaco na área pulmonar e sendo mais nítida na área mitral do que na área tricúspide, associada aos achados radiológicos, levaram à hipótese de discordância atrioventricular e ventriculoarterial (transposição corrigida das grandes artérias). O eletrocardiograma de todos os pacientes mostrou sobrecarga do ventrículo colocado à esquerda. $\mathrm{O}$ vetocardiograma evidenciou rotação anti-horária da alça de despolarização ventricular e deslocada para a esquerda e para trás no plano horizontal. Esses dados, dentro do raci- 


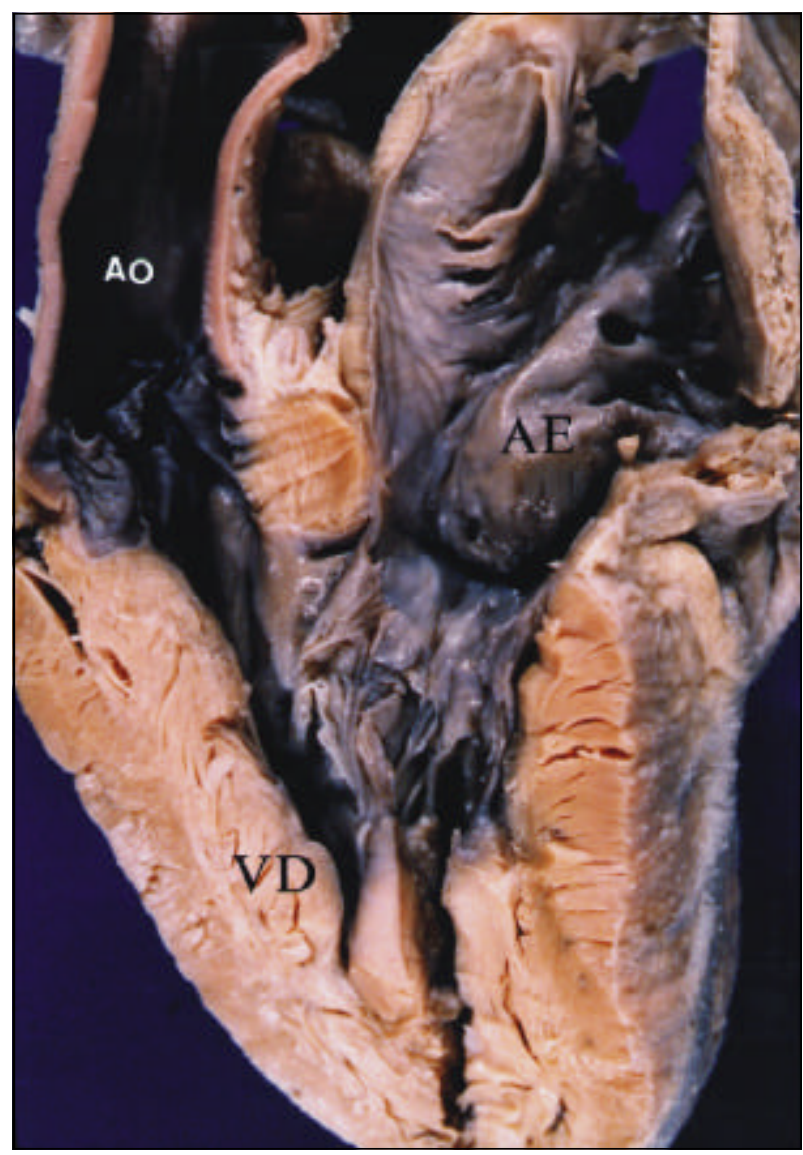

Fig. 3 - Câmaras cardíacas da esquerda abertas (caso ${ }^{\circ} 1$ 1). Nota-se conexão entre átrio morfologicamente esquerdo (AE) e o ventrículo direito (VD), de onde emerge a aorta (Ao).

ocínio de discordância atrioventricular e ventriculoarterial, sugeriram que a câmara ventricular à esquerda era dominante e a da direita pudesse ser hipoplásica. Em todos os casos, o ecocardiograma bidimensional mostrou ausência da conexão atrioventricular direita.

No que diz respeito à nomenclatura, se denominarmos simplesmente de atresia mitral com inversão ventricular, certamente criaríamos uma dificuldade na interpretação da anomalia em questão, visto que, em termos hemodinâmicos,

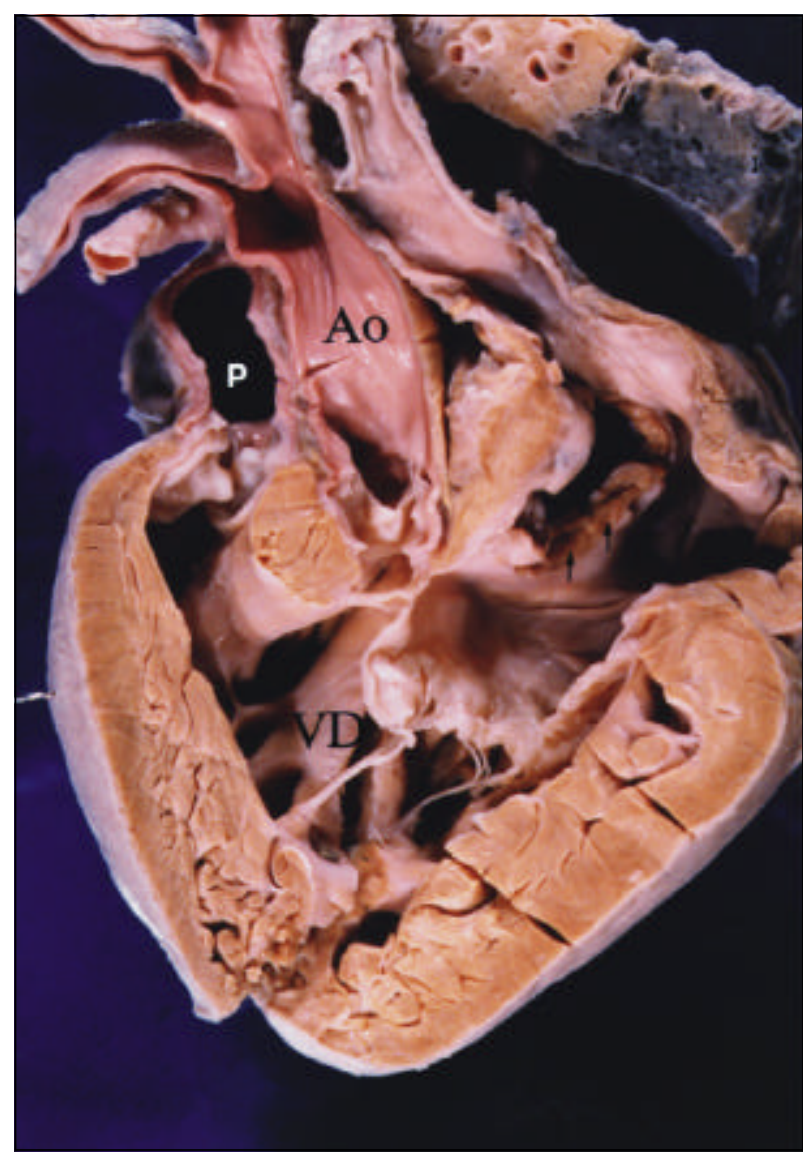

Fig. 4 - Câmaras cardíacas da esquerda abertas (caso nº 2 ). Observa-se no septo atrial orifício de septectomia com bordas cruentas (setas). $\mathrm{O}$ ventrículo dominante é morfologicamente direito (VD) e dele emergem a pulmonar (P) anteriormente e a aorta (Ao).

trata-se de uma atresia tricúspide (ou seja, da valva atrioventricular direita), onde freqüentemente é o VE o ventrículo dominante e a conexão ventriculoarterial do tipo concordante, e o emprego do termo atresia mitral levaria a uma confusão em potencial. Daí a necessidade do uso simplesmente descritivo, isto é, da análise segmentar seqüencial ${ }^{3,4}$. Em relação ao $2^{\circ}$ caso, onde as conexões venosas sistêmica e pulmonar estão dispostas como num situs inversus, a morfologia atrial compatível com isomerismo atrial esquerdo

\begin{tabular}{|c|c|c|c|c|c|}
\hline Paciente & 1 & 2 & 3 & 4 & 5 \\
\hline Idade & $4 \mathrm{~m}$ & $3 a$ & $7 a$ & $11 \mathrm{a}$ & $14 a$ \\
\hline Sexo & M & $\mathrm{F}$ & $\mathrm{M}$ & M & $\mathrm{F}$ \\
\hline Situs & Solitus & Ambíguo & $\begin{array}{c}\text { Solitus } \\
\text { (isomerismo esquerdo) }\end{array}$ & Solitus & Solitus \\
\hline Ápex & Dextro & Dextro & Levo & Levo & Levo \\
\hline Relação V/A & DVSVD & DVSVD & DVSVD & DVSVD & DVSVD \\
\hline Valva Pulmonar & AP & Sem estenose (HP) & EPIV & AP & EP \\
\hline Cirurgia & BT & $\begin{array}{l}\text { Bandagem TP + } \\
\text { Blalock-Hanlon }\end{array}$ & $\begin{array}{c}\text { Cavopulmonar } \\
\text { total }\end{array}$ & $\begin{array}{c}\text { BT 2x } \\
\text { 15dias - 11a }\end{array}$ & $\begin{array}{l}\text { BT } 2 x \\
2 a-6 a\end{array}$ \\
\hline Evolução & Óbito & Óbito & Clínica & Clínica & Clínica \\
\hline Anátomo & Sim & Sim & - & - & - \\
\hline
\end{tabular}


só foi confirmada à necropsia. Todavia, mesmo nesse caso, como nos demais, a melhor descriçãoé, sem dúvida alguma, ausência da conexão atrioventricular à direita, com o átrio à esquerda conectado com o ventrículo morfologicamente direito dominante.

Ainda há que se considerar que termos como atresia mitral e tricúspide, não distinguem entre imperfuração valvar na presença de conexão atrioventricular biventricular e ausência de uma conexão atrioventricular. Corações com ausência de conexão atrioventricular são estruturalmente diferentes daqueles com conexão atrioventricular biventricular. No $1^{\circ}$ caso, não há uma conexão direta entre o átrio em questão e o ventrículo rudimentar e os septos atrial e ventricular se desalinham. Por outro lado, quando há uma valva imperfurada, temos um alinhamento dos septos atrial e ventricular, além da presença de uma conexão direta entre o átrio e o ventrículo através do anel valvar e da valva imperfurada.

Com relação à conexão ventriculoarterial do tipo dupla via de saída do VD, encontrada em todos os nossos casos, está em concordância com o encontrado freqüentemente quando se tem dupla via de entrada de VD dominante, uma vez que o VE rudimentar raramente suporta um vaso arterial. A valva atrioventricular esquerda, em todos os nossos casos, se ligava exclusivamente ao VD dominante e tinha, portanto, morfologia de valva tricúspide. Ho e col ${ }^{5}$ descreveram três casos semelhantes aos nossos, apenas diferindo pelo fato de que a valva atrioventricular esquerda apresentava straddling e overriding, não observados em nenhum dos nossos pacientes.

Shinebourne e col ${ }^{6}$ descreveram 18 casos de conexão atrioventricular univentricular com VD dominante, sendo 12 com dupla via de entrada, dois com ausência de conexão à esquerda e em quatro havia anatomia semelhante aos nossos casos (ausência de conexão atrioventricular à direita) exceto pelo fato de que a aorta estava à direita em dois casos, enquanto que em nossos pacientes a aorta estava sempre à esquerda.

Nosso paciente que apresentava isomerismo atrial esquerdo foi o único sem estenose pulmonar, fato este em concordância com a configuração mais freqüentemente encontrada no isomerismo atrial esquerdo e raro no isomerismo atrial direito.

Quatro dos nossos pacientes foram considerados para tratamento cirúrgico, sendo que um deles se submeteu a anastomose cavopulmonar. Sem dúvida, a derivação atriopulmonar representa a melhor opção de tratamento para esses pacientes, apesar dos inconvenientes de uma cirurgia de Fontan em corações com conexão atrioventricular univentricular com VD dominante ${ }^{7}$.

Finalmente, chamamos ainda a atenção para o fato da semelhança morfológica existente entre estes cinco casos, o que possibilita sem dúvida alguma a sua suspeição diagnóstica através dos elementos clínicos já citados.

\section{Referências}

1. Casta A, Wolf WJ, Sapire DW - Absent right atrioventricular connexion with the left atrium connected to the morphologically right ventricle, a right-sided rudimentary left ventricle, and right juxtaposition of the atrial appendages: documentation by angiography and cross-sectional echocardiography. Int $\mathrm{J}$ Cardiol 1985; 7: 21-6.

2. Ebaid M, Cavalini JF, Aiello VD - Absent right atrioventricular connection with the left atrium connected to a left-sided dominant right ventricle - anatomical and functional aspects in four cases. Cardiol Young 1994; 4: 381-5.

3. Anjos R, Murdoch I, Qureshi S - Absence of one atrioventricular connexion - a terminologic problem. Int J Cardiol 1991; 30: 239-42.
4. Anderson RH, Tynan M - Description of complex forms of atrioventricular valvar atresia. Int J Cardiol 1991; 30: 243-7.

5. Ho SY, Milo S, Anderson RH et al - Straddling atrioventricular valve with absent atrioventricular connection. Report of 10 cases. Br Heart J 1982; 47: 344-52.

6. Shinebourne EA, Lau KC, Calcaterra G, Anderson RH - Univentricular heart of right ventricular type: clinical, angiographic and electrocardiographic features. Am J Cardiol 1980; 46: 439-45.

7. Matsuda H, Kawashima Y, Kishimoto H et al - Problems in the modified Fontan operation for univentricular heart of the right ventricular type. Circulation 1987; 76(suppl III): III 45-III 52. 